\title{
Personalizing Suicidology
}

\author{
Craig J.R. Sewall ${ }^{1}$ and Aidan G.C. Wright ${ }^{2}$ \\ 'Department of Psychiatry, University of Pittsburgh, PA, USA \\ ${ }^{2}$ Department of Psychology, University of Pittsburgh, PA, USA
}
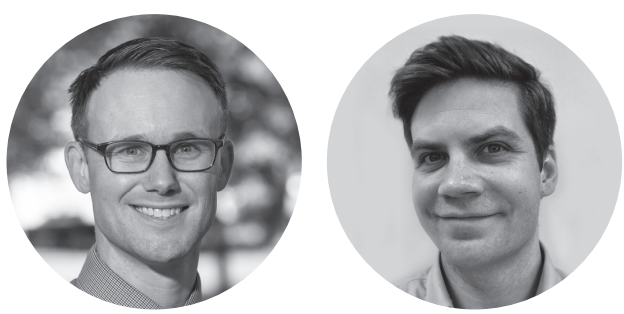

It, ultimately, is the unique and individual suicidal case, intensively studied, that provides the true understanding of suicidal behavior. As we search desperately for commonalities, it is only through the idiographic method that we can remind ourselves of the complexity of the suicidal character.

Alan Berman (2003, p. 200)

What makes people suicidal? Unfortunately, despite a century of suicide research and theorizing, the field of suicidology has yet to provide a sufficient answer to this foundational question. We have managed to identify a multitude of empirically and theoretically derived risk factors - spanning everything from social forces at the societal level (e.g., Durkheim, 1897/1951) to biological mechanisms at the microscopic level (e.g., Mann, 2013; Pedersen et al., 2012), and everywhere in between (see Turecki et al., 2019) - but "there is no evidence that any known risk factors - broad or specific - approach what many might define as clinical significance" (Franklin et al., 2016, p. 215). The inability of single or small sets of risk factors to adequately predict suicide risk has reinforced the highly complex nature of suicidal thoughts and behaviors (STBs) and prompted the use of machine-learning methods, which are better suited to model the types of complex dynamics that may be necessary to predict risk (Franklin et al., 2016; Ribeiro et al., 2016).

Initial attempts at applying machine learning to suicide risk prediction have appeared to support this assertion, as machine-learning approaches have improved predictive accuracy and identified novel risk factors (Burke et al., 2019). However, despite these advances, our ability to predict short-term risk for suicide remains poor (Belsher et al., 2019; Burke et al., 2019) - which is a crucial impediment to effective suicide intervention - and there is evidence that, due to methodological oversights, the predictive performance of machine-learning algorithms in recent suicide research is not as impressive as advertised (Jacobucci et al., 2021). While we share in the enthusiasm for machine learning and the promise it holds for capturing the complexity inherent in STBs and agree with calls to improve and advance machine-learning applications in su- icide research (Burke et al., 2019; Jacobucci et al., 2021), we believe that efforts at suicide prediction will continue to fall short because of a fundamental misalignment between how STBs are typically studied and how they inherently emerge.

The past half-century of contemporary suicide research has predominantly used nomothetic approaches - which aggregate data across individuals, resulting in omnibus model estimates reflecting group-based or between-person (i.e., inter-individual) averages - as opposed to idiographic approaches, which use person-specific models unique to each individual. We maintain that STBs emerge as a result of a complex interplay among ensembles of contextualized dynamic processes that are highly specific to the individual and, thus, the nomothetic methods traditionally used to study and predict STBs are ill-suited to capture the idiographic data-generating process inherent to suicide. Accordingly, we call for the field of suicidology to prioritize a quantitative idiographic (i.e., personalized) approach to suicide research that, thanks to recent technological advances, is now feasible. We contend that a personalized modeling approach to suicidology represents a superior match between the phenomenon we are studying and the methods used to understand it.

\section{Suicide as a Personalized Dynamic Process}

Suicide is a highly personalized phenomenon. What could be more personal than the prospect of ending the self? Expanding on our thesis presented in italics above, STBs emerge as a result of the interaction between dynamic processes. Note that the apparent redundancy in these words is intentional, as various processes may interact to confer suicide risk, and the way that these processes interact to confer risk may itself change over time. Hence, the processes themselves are dynamic. Accordingly, these dynamic processes are contextualized, in that they occur 
within (and a result of) an individual's context, which may comprise biological, social, economic, geographic, and/ or psychological components. Indeed, it is changes in an individual's context that may explain why the processes leading to STBs change over time. Lastly, but perhaps most importantly, these contextualized dynamic processes are highly specific to the individual. That is, the particular dynamics and contextualized factors that instigate STBs for a certain individual may have a drastically different effect for other individuals. In other words, borrowing the language of Nesselroade (1991), STBs manifest as a result of intra-individual processes, and there are inter-individual differences in these intra-individual processes.

However, nomothetic analyses provide information only about inter-individual processes (i.e., between-person variation), which cannot be applied at the level of the individual (i.e., intra-individual or within-person processes) unless rigorous conditions are met. These conditions, as explicated by the theorems of ergodicity (see Molenaar, 2004; Molenaar \& Campbell, 2009), stipulate that for an analysis of inter-individual variation to produce the same results as an analysis of intra-individual variation, (1) the same statistical model should apply to all the participants in the population (i.e., the population must be homogenous), and (2) the data must have invariant statistical properties over time, such as constant mean and variance (i.e., the data must be stationary). The types of phenomena studied across the so-called soft sciences very rarely meet these strict conditions and, as detailed below, this is particularly true of STBs.

\section{Suicidology and the Curse of Non-Ergodicity}

The statistical theorems of ergodicity date back nearly 100 years. Originally developed to deal with problems germane to statistical physics, the implications of the ergodic theorems for behavioral and psychological sciences were not well understood until introduced by Molenaar and colleagues (Molenaar, 2004; Molenaar \& Campbell, 2009). Although oft-derided as a "soft" science on account of general lack of robust theoretical and replicatory work, the study of human psychological and/or behavioral processes is quite hard (i.e., difficult). People can be thought of as an "integrated dynamic system of behavioral, emotional, cognitive, and other psychological processes evolving over time and space," (Molenaar \& Campbell, 2009, p. 112). In other words, human beings are very complex systems; and there is arguably not a more complex behavioral-psychological process than suicide. Due in part to the fact that STBs are rare events, the dominant quantitative approach in suicide research has been to collect data from lots of people and apply nomothetic analyses, with the assumption that pooling information across multiple people would lead to the discovery of general commonalities or "truths" about how and/or why STBs emerge, which could then be used to describe the majority of people in the population. Unfortunately, when it comes to STBs (as well as most other human behavioral or psychological phenomena) this assumption does not hold. As elucidated by Molenaar and Campbell (2009, p. 112),

It might seem evident that inferences about the state of affairs at the population level constitute general findings that apply to each individual subject in the population. However, applying the findings obtained by pooling across subjects to a single individual in the population involves a shift in level - namely, from the level of interindividual variation to that of intraindividual variation in time and place. Is this shift between levels valid? It will be shown that, generally speaking, the answer is no.

The first condition of ergodicity stipulates that the same statistical model should apply to all individuals in the population. This means that the "main features of a statistical model describing the data are the same across subjects" (Molenaar \& Campbell, 2009, p. 113). In the case of STBs, this suggests that (1) the phenomenology of STBs exhibit the same (or, realistically, very similar) functional form across individuals, (2) the risk factors for STBs exhibit the same (or similar) sign and magnitude across individuals, and (3) the measures of STBs have the same (or similar) psychometric features across individuals. However, STBs are highly heterogeneous in their manifestation - the systems that give rise to STBs may vary substantially from one person to the next (Bernanke et al., 2017; Kleiman et al., 2019; Mou et al., 2020; Niculescu et al., 2017). Even when attempts at homogenization are made by partitioning the population into subgroups based on age, gender, ethnicity, suicide history, etc. - heterogeneity abounds. There is no evidence that any one risk factor is either necessary or sufficient for STBs to occur or recur. In this way, the systems giving rise to STBs exhibit both equifinality - in that a wide range of different factors can lead to STBs - as well as multifinality, such that the same set of factors does not necessarily lead to STBs (cf. Fried \& Robinaugh, 2020).

Examples of equifinality and multifinality in suicide research are plentiful. For multifinality, take for example the oft-cited (though contentious) claim that over $90 \%$ of those who die by suicide had an identifiable psychiatric disorder before death (Turecki \& Brent, 2016). Yet, the overwhelming majority of people with psychiatric disorders do not die by suicide, much less even attempt suicide. Furthermore, even thinking about killing yourself is not a sufficient condition for suicidal behavior, as the majority 
of those with suicidal ideation do not and will not attempt suicide. Equifinality, on the other hand, is exemplified by the massive assortment of identified risk factors that are associated with STBs. Interview 10 different people with suicidal ideation or a recent suicide attempt and you may well find 10 different reasons for their suicidality.

The second condition of ergodicity is a type of homogeneity over time, known as "stationarity," which stipulates that the main features of a statistical model describing the data should be invariant across time (Molenaar, 2004). That is, the associations among variables characterizing the manifestation of STBs should not change as a function of time. This means that, for instance, in a study of suicidal individuals spanning 12 months, the sign and magnitude of the association between suicidal behavior and thwarted belongingness (or emotional dysregulation, or interpersonal distress, etc.) should be the same during Month 1 as it is during Month 12. However, as detailed above, variation in a person's context - such as changes in weather, job/ school status, income, substance use, treatment regiment, or social network - will frequently cause the relevance and potency of certain risk factors to fluctuate within individuals over time.

The non-stationarity and non-homogeneity (i.e., the non-ergodicity) of STBs makes prediction extremely difficult - a task that is nigh impossible with the stability assumptions inherent in nomothetic approaches that have dominated suicidology in recent history. As aptly put by Berman (2003, p. 198):

Much has been learned from and much has been gained by this nomothetic approach...Yet, as valuable and important as these studies are, you might ask "Of what use are they?", and "How do I apply, clinically, this mass of aggregated data?". Typically, these studies paint with such a broad brush that the caregiver, who is charged with translating researchers' findings into clinical application, is potentially and inadvertently misled. For example: If diagnosed mental disorders are necessary conditions for suicidal behavior, and I work in a setting where all adolescents have diagnosed mental disorders, what then? If we have learned that $50 \%$ of suicidal females have made a prior suicide attempt, then $50 \%$ have not. Which of these is a risk factor?

\section{Implications for Suicide Research}

The ergodicity problem of STBs has major implications for the field of suicidology, as most suicide research over the past half-century has depended on nomothetic analyses to identify risk factors, inform theories and policy, as well as develop and test interventions. While these analyses have yielded important findings, the non-ergodic nature of STBs implies that the information gained from these nomothetic analyses cannot be dependably applied at the intra-individual level. Since STBs manifest as a result of contextualized intra-individual processes that are highly heterogeneous between-persons and non-stationary within-persons, using nomothetic analyses to identify risk factors, develop interventions, and inform theory will produce results that will adequately apply to only a small portion of the population over a limited period of time. Consequently, our prediction models can passably identify who may be at elevated risk of suicide - an inter-individual phenomenon - but are unable to identify when a person may be at elevated risk - an intra-individual phenomenon. This inability to identify when a person is at risk for suicide has made it challenging to deliver interventions to people when they are at highest risk. The mismatch between nomothetic analyses with the person-specific manifestation of STBs also contributes to our suicide theories being either too broad - so as to capture as many of the heterogeneous manifestations of STBs as possible - or too narrow, resulting in theories that passably describe the suicidal process for some but not for most. Similarly, policies and interventions deemed "evidence based" or "best practice" via nomothetic approaches might not be effective for the majority of suicidal individuals, or more concerningly, may even be harmful for some.

\section{Idiographic Methods Are Necessary to Capture the Personalized Dynamics of Suicide}

We contend that if the field of suicidology continues to conduct research by employing nomothetic statistical methods fundamentally incapable of capturing the personalized data-generating process inherent to the manifestation of STBs, then our ability to understand, predict, and prevent suicide will remain stagnant. Simply put, to sufficiently capture a process that is largely person-specific requires person-specific (i.e., idiographic) methodology.

Now, calls for the field of suicidology to prioritize the idiographic approach to studying STBs are by no means new (e.g., see Berman, 2003; Leenaars, 2002). Suicidology has a noteworthy history of idiographic research, which has typically involved qualitative analyses of case studies, suicide notes, clinical interviews, or psychological autopsies (Leenaars, 2002). However, given the time and emotional demands inherent to most qualitative methods (e.g., clinical interviews), it is extremely difficult, if not impossible, to carry out longitudinal qualitative work over an intensive timeframe. As a result, when longitudinal qualitative methods are used, the intervals between 
interviews are expansive, obliging researchers to rely on retrospective reports from participants, which are prone to recall bias.

The quantitative idiographic approach, however, is capable of collecting dense, high-dimensional data in a way that can adequately capture the personalized intra-individual processes that give rise to STBs. In this approach, rather than fitting a collection of individuals to a statistical model, statistical models are fit to the individuals - that is, the individual's system of time-varying variables is investigated across time (Molenaar \& Campbell, 2009). Admittedly, this kind of person-specific approach is challenging, as a wide assortment of data must be collected from individuals at an intensive timeframe, and computational methods are required that can adequately manage and analyze these dense data. Fortunately, recent technological advances have made it possible to pursue a quantitative idiographic approach to studying STBs in a way that is feasible, affordable, scalable, and of minimal burden to participants. Ambulatory assessment capitalizes on the ubiquity of smartphones and wearable devices to collect ecologically valid data on an array of variables multiple times a day (or even in real time). These data may be collected in an active manner - in the form of ecological momentary assessments - or in a passive manner, in the form of sensor data automatically tracked by participants' mobile devices. And concomitant computational developments make it possible to readily track, upload, store, process, and analyze these intensive data.

Researchers in the psychological-behavioral sciences have already started to harness the quantitative idiographic approach to develop personalized models of psychopathology (e.g., see Wright \& Woods, 2020) as well as personalized treatment models (Fisher \& Boswell, 2016; Rubel et al., 2018), leading to innovations in how psychopathology is understood, predicted, and treated. We believe that a personalized modeling approach to STBs holds substantial promise and should be a prioritized avenue of suicide research, as it provides a modeling framework that is consistent with the complex idiographic processes inherent to suicide risk and is well-suited to detect person-specific patterns of risk factors that could enhance suicide prediction, theory, and prevention.

\section{Promising Quantitative Idiographic Approaches to Suicide Research}

There are a variety of potential quantitative idiographic avenues to suicide research; we highlight a few that hold promise for improving our empirical and theoretical insight into STBs.
Although the processes that give rise to STBs are highly personalized, this does not necessarily mean that each individual's manifestation is completely unique. Indeed, recent suicide research has identified specific subgroups of individuals who share certain commonalities in how STBs emerges (Bernanke et al., 2017; Niculescu et al., 2017). However, as opposed to nomothetic analyses, which search for commonalities by aggregating across people and/or time (i.e., a top-down approach), the quantitative idiographic approach fits statistical models to each person individually and then searches for commonalities among the person-specific results (i.e., a bottom-up approach). In this way, the idiographic approach to identifying commonalities remains consistent with the personalized data-generating process inherent to STBs, but also allows researchers to see whether or to what extent person-specific processes are similar across people.

One promising bottom-up approach is group iterative multiple model estimation (GIMME; Gates et al., 2017; Gates \& Molenaar, 2012; Lane et al., 2019), a data-driven approach for identifying complex patterns of relations shared across heterogenous time series. Although originally developed for use on functional brain imaging data, GIMME is now being applied to psychological-behavioral time-series data. For instance, Wright and colleagues (Woods et al., 2020; Wright et al., 2019) used GIMME to investigate idiographic commonalities in the affective and interpersonal processes among those with borderline personality disorder. GIMME is a particularly promising approach as it fits individual-level, group-level, and subgroup-level models (Gates et al., 2017; Henry et al., 2019), making it well equipped to compare the idiographic versus nomothetic manifestation of STBs and identify potential (sub)groupings that could help inform theory or interventions. Furthermore, by automating this process, GIMME takes the laborious work of idiographic analyses - fitting person-specific models across dozens or hundreds of individuals is no easy task - and makes it scalable to large samples.

Personalized modeling of STBs via a quantitative idiographic approach has the potential to improve suicide theory considerably. Given the heterogeneity of how suicide arises across individuals, the prospect of a grand theory that precisely and parsimoniously explains all these different manifestations is quite unlikely. Rather, it is more plausible that certain theories do better at explaining different individual's STBs manifestation process. For instance, some individuals may experience STBs when feelings of defeat and entrapment are elevated (O'Connor \& Kirtley, 2018), while for others STBs may be triggered by feelings of burdensomeness and thwarted belongingness (van Orden et al., 2010). Furthermore, the idiographic approach may motivate the adaptation of current theories - for ex- 
ample, if an individual's STBs emerge as a result of feelings of thwarted belongingness and entrapment - as well as the development of new theories. With the personalized approach, it would be possible to match the data-generating process dictated by certain suicide theories with the observed data from suicidal individuals to identify which theories best capture the suicide-generating process for which individuals.

\section{Conclusion}

Since the dawn of modern statistics, suicidologists have been employing standard group-level analyses as the predominant quantitative approach to understanding and predicting STBs. Because STBs emerge as a result of processes that are highly contextualized to the individual, employing these nomothetic analyses to understand what in actuality is an idiographic process is akin to using satellite imagery to understand the behavior of microbes. In this editorial, we have illustrated how a personalized modeling approach (i.e., quantitative idiographic analyses), made possible by developments in ubiquitous computing and computational modeling, represents a better alignment with the idiographic data-generating process inherent to the manifestation of STBs, and, thus, holds substantial promise for improving our theory, predictions, and interventions.

\section{References}

Belsher, B. E., Smolenski, D. J., Pruitt, L. D., Bush, N. E., Beech, E. H., Workman, D. E., Morgan, R. L., Evatt, D. P., Tucker, J., \& Skopp, N. A. (2019). Prediction models for suicide attempts and deaths: A systematic review and simulation. JAMA Psychiatry, 76(6), 642651. https://doi.org/10.1001/jamapsychiatry.2019.0174

Berman, A. L. (2003). An idiographic approach to understanding suicide in the young. In R. A. King \& A. Apter (Eds.), Suicide in children and adolescents (pp. 198-210). Cambridge University Press.

Bernanke, J. A., Stanley, B. H., \& Oquendo, M. A. (2017). Toward finegrained phenotyping of suicidal behavior: The role of suicidal subtypes. Molecular Psychiatry, 22(8), 1080-1081. https://doi. org/10.1038/mp.2017.123

Burke, T. A., Ammerman, B. A., \& Jacobucci, R. (2019). The use of machine learning in the study of suicidal and non-suicidal self-injurious thoughts and behaviors: A systematic review. Journal of Affective Disorders, 245, 869-884. https://doi.org/10.1016/j. jad.2018.11.073

Durkheim, E. (1951). Suicide: A study in sociology (J.A. Spaulding \& G. Simpson, Trans.). The Free Press. (Original work published 1897)

Fisher, A. J., \& Boswell, J. F. (2016). Enhancing the personalization of psychotherapy with dynamic assessment and modeling. Assessment, 23(4), 496-506. https://doi.org/10.1177/ 1073191116638735
Franklin, J. C., Ribeiro, J. D., Fox, K. R., Bentley, K. H., Kleiman, E. M., Huang, X., Musacchio, K. M., Jaroszewski, A. C., Chang, B. P., \& Nock, M. K. (2016). risk factors for suicidal thoughts and behaviors: A meta-analysis of 50 years of research. Psychological Bulletin, 143(2), 187-232. https://doi.org/10.1037/bul0000084

Fried, E. I., \& Robinaugh, D. J. (2020). Systems all the way down: Embracing complexity in mental health research. BMC Medicine, 18, 205. https://doi.org/10.1186/s12916-020-01668-w

Gates, K. M., Lane, S. T., Varangis, E., Giovanello, K., \& Guiskewicz, K. (2017). Unsupervised classification during time-series model building. Multivariate Behavioral Research, 52(2), 129-148. https://doi.org/10.1080/00273171.2016.1256187

Gates, K. M., \& Molenaar, P. C. M. (2012). Group search algorithm recovers effective connectivity maps for individuals in homogeneous and heterogeneous samples. Neurolmage, 63(1), 310-319. https://doi.org/10.1016/j.neuroimage.2012.06.026

Henry, T. R., Feczko, E., Cordova, M., Earl, E., Williams, S., Nigg, J. T., Fair, D. A., \& Gates, K. M. (2019). Comparing directed functional connectivity between groups with confirmatory subgrouping GIMME. Neurolmage, 188, 642-653. https://doi.org/10.1016/j. neuroimage.2018.12.040

Jacobucci, R., Littlefield, A. K., Millner, A. J., Kleiman, E. M., \& Steinley, D. (2021). Evidence of inflated prediction performance: A commentary on machine learning and suicide research. Clinical Psychological Science. https://doi.org/10.1177/ 2167702620954216

Kleiman, E. M., Glenn, C. R., \& Liu, R. T. (2019). Real-time monitoring of suicide risk among adolescents: Potential barriers, possible solutions, and future directions. Journal of Clinical Child and Adolescent Psychology, 48(6), 934-946. https://doi.org/10.1080/ 15374416.2019.1666400

Lane, S. T., Gates, K. M., Pike, H. K., Beltz, A. M., \& Wright, A. G. C. (2019). Uncovering general, shared, and unique temporal patterns in ambulatory assessment data. Psychological Methods, 24(1), 54-69. https://doi.org/10.1037/met0000192

Leenaars, A. A. (2002). In defense of the idiographic approach: Studies of suicide notes and personal documents. Archives of Suicide Research, 6(1), 19-30. https://doi.org/10.1080/1381111 0213125

Mann, J. J. (2013). The serotonergic system in mood disorders and suicidal behaviour. Philosophical Transactions of the Royal Society of London. Series B, Biological Sciences, 368(1615), 20120537. https://doi.org/10.1098/rstb.2012.0537

Molenaar, P. C. M. (2004). A manifesto on psychology as idiographic science: Bringing the person back into scientific psychology, this time forever. Measurement, 2(4), 201-218. https://doi. org/10.1207/s15366359mea0204_1

Molenaar, P. C. M., \& Campbell, C. G. (2009). The new person-specific paradigm in psychology. Current Directions in Psychological Science, 18(2), 112-117. https://doi.org/10.1111/j.1467-8721. 2009.01619.x

Mou, D., Kleiman, E. M., \& Nock, M. K. (2020). Proposed directions for suicide research: Incorporating successful approaches from other disciplines. British Journal of Psychiatry, 217(6), 659-660. https://doi.org/10.1192/bjp.2020.58

Nesselroade, J. R. (1991). Interindividual differences in intraindividual change. In L. M. Collins \& J. L. Horn (Eds.), Best methods for the analysis of change: Recent advances, unanswered questions, future directions (pp. 92-105). American Psychological Association. https://doi.org/10.1037/10099-006

Niculescu, A. B., Le-Niculescu, H., Levey, D. F., Phalen, P. L., Dainton, H. L., Roseberry, K., Niculescu, E. M., Niezer, J. O., Williams, A., Graham, D. L., Jones, T. J., Venugopal, V., Ballew, A., Yard, M., Gelbart, T., Kurian, S. M., Shekhar, A., Schork, N. J., Sandusky, G. E., \& Salomon, D. R. (2017). Precision medicine for suicidality: From universality to subtypes and personalization. Mo- 
lecular Psychiatry, 22(9), 1250-1273. https://doi.org/10.1038/ mp.2017.128

O'Connor, R. C., \& Kirtley, O. J. (2018). The integrated motivational-volitional model of suicidal behaviour. Philosophical Transactions of the Royal Society B: Biological Sciences, 373(1754), 20170268. https://doi.org/10.1098/rstb.2017.0268

Pedersen, M. G., Mortensen, P. B., Norgaard-Pedersen, B., \& Postolache, T. T. (2012). Toxoplasma gondii infection and selfdirected violence in mothers. Archives of General Psychiatry, 69(11), 1123-1130. https://doi.org/10.1001/archgenpsychiatry. 2012.668

Ribeiro, J. D., Franklin, J. C., Fox, K. R., Bentley, K. H., Kleiman, E. M., Chang, B. P., \& Nock, M. K. (2016). Letter to the Editor: Suicide as a complex classification problem: Machine learning and related techniques can advance suicide prediction - a reply to Roaldset (2016). Psychological Medicine, 46(9), 2009-2010. https://doi. org/10.1017/S0033291716000611

Rubel, J. A., Fisher, A. J., Husen, K., \& Lutz, W. (2018). Translating person-specific network models into personalized treatments: Development and demonstration of the dynamic assessment treatment algorithm for individual networks (DATA-IN). Psychotherapy and Psychosomatics, 87(4), 249-251. https://doi.org/ 10.1159/000487769

Turecki, G., \& Brent, D. A. (2016). Suicide and suicidal behaviour. The Lancet, 387(10024), 1227-1239. https://doi.org/10.1016/ S0140-6736(15)00234-2

Turecki, G., Brent, D. A., Gunnell, D., O'Connor, R. C., Oquendo, M. A., Pirkis, J., \& Stanley, B. H. (2019). Suicide and suicide risk. Nature Reviews Disease Primers, 5(1). https://doi.org/10.1038/s41572019-0121-0

van Orden, K. A., Witte, T. K., Cukrowicz, K. C., Braithwaite, S. R., Selby, E. A., \& Joiner, T. E. (2010). The interpersonal theory of suicide. Psychological Review, 117(2), 575-600. https://doi.org/10.1037/ a0018697

Woods, W. C., Arizmendi, C., Gates, K. M., Stepp, S. D., Pilkonis, P. A., \& Wright, A. G. C. (2020). Personalized models of psycho- pathology as contextualized dynamic processes: An example from individuals with borderline personality disorder. Journal of Consulting and Clinical Psychology, 88(3), 240-254. https://doi. org/10.1037/ccp0000472

Wright, A. G. C., Gates, K. M., Arizmendi, C., Lane, S. T., Woods, W. C., \& Edershile, E. A. (2019). Focusing personality assessment on the person: Modeling general, shared, and person specific processes in personality and psychopathology. Psychological Assessment, 31(4), 502-515. https://doi.org/10.1037/pas0000617

Wright, A. G. C., \& Woods, W. C. (2020). Personalized models of psychopathology. Annual Review of Clinical Psychology, 16(1), 1-26. https://doi.org/10.1146/annurev-clinpsy-102419-125032

Published online November 16, 2021

\section{Craig J. R. Sewall}

Department of Psychiatry

University of Pittsburgh

Pittsburgh, PA

USA

CJS227@pitt.edu

Craig Sewall, PhD, is a postdoctoral fellow at the Department of Psychiatry at the University of Pittsburgh, PA, USA, and a licensed clinical social worker. His research focuses on harnessing advancements in quantitative modeling and ubiquitous computing to improve our understanding of, and ability to predict, suicidal thoughts and behaviors.

Aidan Wright, PhD, is an associate professor of psychology at the University of Pittsburgh, PA, USA. His research focuses on the interface of personality and psychopathology, with a particular emphasis on studying these phenomena in life as it is lived, using ambulator assessment techniques in naturalistic settings. 